\title{
Gene expression profile of Vitamin D3 treated HL60 cells shows an incomplete molecular phenotypic conversion to monocytes
}

\author{
E Tagliafico ${ }^{1}$, E Tenedini ${ }^{1}$, A Bergamaschi ${ }^{1}$, R Manfredini ${ }^{1}$, \\ R Percudani ${ }^{2}$, M Siena ${ }^{1}$, T Zanocco-Marani ${ }^{1}$, A Grande $^{1}$, \\ M Montanari ${ }^{1}$, C Gemelli ${ }^{1}$, U Torelli ${ }^{1}$ and S Ferrari ${ }^{\star,}, 1$ \\ 1 Università di Modena e Reggio Emilia, Dipartimento di Scienze Biomediche, \\ Sezione di Chimica Biologica, Via Campi, 287, 41100, Modena, Italy \\ 2 Università di Parma, Dipartimento di Biochimica e Biologia Molecolare, Viale \\ delle Scienze, 43100 Parma, Italy \\ * Corresponding author: Sergio Ferrari. Tel: +39-059-2055400 \\ Fax: +39-059-2055410; E-mail: ferrari.sergio@unimo.it
}

Received 29.3.02; revised 11.6.02; accepted 11.7.02

Edited by M Piacentini

\begin{abstract}
By high density oligonucleotide microarrays we have studied the expression profile of proliferating and VD treated HL60 cells and the molecular phenotype of VD monocytes and that of CD14+ peripheral monocytes has been compared. The results indicate that important changes in functional categories of the differentially expressed genes underlie the differentiation transition from myeloblasts to monocytes. This differential gene expression pattern leads to an increased expression of mRNAs involved in surface and external activities since many of the VD induced genes belong to ligand binding, receptors, cell surface antigens, defense/ immunity and adhesion molecules functional categories. The results also indicate that the molecular phenotypes of monocytes and VD induced cells diverge for a small but significant set of defense related genes. Particularly, class II MHC genes are not expressed in these cells. Furthermore, the high levels of expression of these genes induced by serum treatment of monocytes are decreased by VD.
\end{abstract}

Cell Death and Differentiation (2002) 9, 1185-1195. doi:10.1038/sj.cdd.4401104

Keywords: cell differentiation; oligonucleotide microarrays; myeloid cells

Abbreviations: AML, acute myeloid leukemia; ATRA, all-trans retinoic acid; avg. diff., average difference; DMSO, dimethylsulphoxide; DMT, data mining tool; FAB, French-American-British Classifiaction; FBS, fetal bovine serum; GO, gene ontology; IMDM, Iscove's modified Dulbecco's medium; MGG. May-Grünwald and Giemsa; MAS 4.0, microarray analysis suite 4.0; MHC, major histocompatibility complex; PBMC, peripheral blood mononuclear cells; RT-PCR, reverse transcription-polymerase chain reaction; VD, $1, \alpha 25$ dihydroxivitamin D3

\section{Introduction}

One of the most peculiar aspects of leukemic myelopoiesis is the arrest of the differentiation process which occurs usually at different, although always very early, stages of myeloid maturation. ${ }^{1,2}$ This certainly reflects the well known condition of AML blast cells which are characterized by a conspicuous number of genetic abnormalities, ${ }^{3}$ mainly sporadic or recurrent and less frequently specific. In spite of the large variety of genetic abnormalities, these cells are constantly characterized by their inability to proceed spontaneously toward terminal differentiation. ${ }^{4}$ Even myeloid growth factors mainly stimulate the precursor proliferation, but in no way induce the differentiation pathway. ${ }^{5,6}$ This is one of the main reasons of interest for the use of pharmacological agents, such as all trans-retinoic acid (ATRA), Dimethylsulphoxide (DMSO), Butyrate derivatives, and Vit. D3 (VD), able to activate, in vitro and in vivo, a differentiation program. ${ }^{7}$ These differentiating agents are able to induce a phenotypic conversion of $\mathrm{M} 2$ and $\mathrm{M} 3$ blast cells, i.e. leukemic myeloblasts as defined by $F A B$ and immunophenotypic classification of AML, ${ }^{1,2}$ to a more advanced stage of maturation. ${ }^{8-11}$ There are, however, few indications about the correspondence between the pharmacologically induced molecular phenotype and the physiologically differentiated cell. We do not know whether the genetic program activated by these agents is similar to that occurring in normal myelopoiesis. To tackle this problem, we studied the changes occurring in the gene expression profile during the differentiation of HL60 cells treated with VD using the Affymetrix GeneChip technology. ${ }^{12-14}$ Because of the lack of systematic functional annotation for human genes, we have extended the Affymetrix Human Genome U95A (HGU95A) data set with a controlled vocabulary of biological annotations provided by the Gene Ontology (GO) $)^{15,16}$ consortium that includes three extensive ontologies describing molecular function, biological process and cellular component that can be used to describe gene products in any organisms. Therefore, our approach has been based on the characterization of the differentially expressed genes according to selected GO categories. Furthermore, the molecular phenotype of VD treated HL60 cells has been compared to that of peripheral blood monocytes. Our results indicate that VD treated HL60 cells express genes associated with the mechanisms of natural immunity with the important exception concerning class II MHC genes that are not expressed in these cells, whereas they are clearly expressed in mature peripheral monocytes. Furthermore the VD treated HL60 cells express a limited set of specific genes not expressed in normal monocytes or in activated macrophages. ${ }^{17-19}$ This suggests that VD activates a genetic program which is partially different from that leading to normal peripheral monocytes. Finally VD inhibits the increased 
expression of class II MHC genes in CD14+ monocytes activated by serum.

\section{Results}

\section{Abundance and complexity of mRNAs based on gene expression profile of proliferating and VD treated HL60 cells}

The comparison of mRNAs abundance between proliferating and VD treated HL60 cells indicates that no difference in the distribution of mRNA abundances is detectable (data not shown).

Out of 12600 genes present in the human genome U95A (HGU95A) GeneChip array, 5115 (40.5\%) are expressed in proliferating HL60 cells and 4825 (38.3\%) in VD treated cells. The number of different mRNA sequences, i.e. sequence complexity, is therefore very similar in proliferating and differentiated cells (Figure 1).

This figure also shows that about $90 \%$ of the genes are expressed in both cellular contexts, and among them, about $30 \%$ are modulated. Six hundred and forty-one mRNAs are decreased in VD treated cells and 61 of them are completely down regulated and therefore specific of proliferating cells. On the contrary, 412 genes have an increased expression in differentiated HL60 cells, and among them 115 are induced and therefore specific of VD treated cells. Therefore, the large majority of the expressed genes (3271) is substantially unchanged. All these figures have been obtained by elaborating the results of three independent experiments, and we believe that they reliably reflect the genome expression in our cellular contexts. This study did not further consider 730 genes in proliferating and 382 genes in VD treated cells because

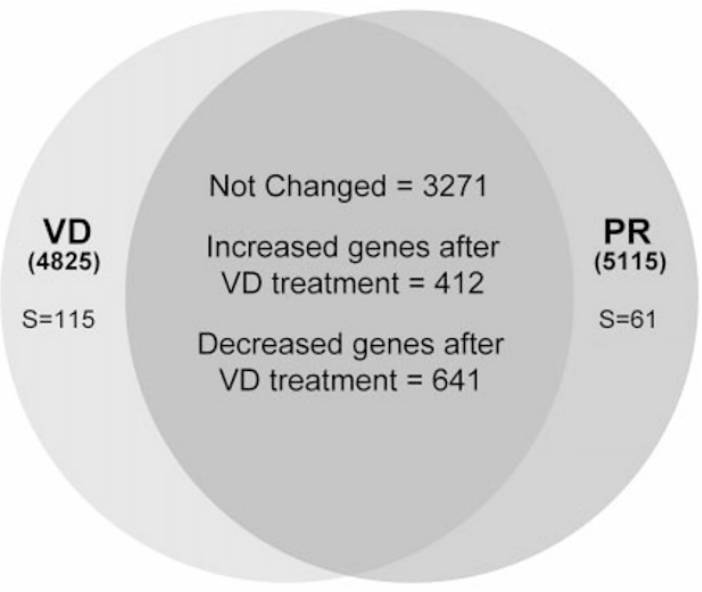

Figure 1 The diagram shows the overall number of genes present in the two types of cells, proliferating (PR) and differentiated (VD) HL60 cells, subdivided according to their relative abundance. Three independent experiments have been performed and only the genes called present, based on the absolute call parameter, in three out of three experiments were recorded. The term 'specific' (S) indicates those genes whose expression was present only in one type of cell and absent in the other type. Decreased and increased indicate changes in genes expression occurring in VD treated cells they are scarcely expressed sequences near the detection threshold.

\section{Biological characterization of the global gene expression profile in proliferating and differentiated HL60 cells}

In order to improve the understanding of the biological meaning of microarrays data, we enriched the list of gene names and the descriptions of the HGU95A data set using a controlled vocabulary of biological annotations provided by the Gene Ontology (GO) consortium. ${ }^{15,16}$

The GO vocabulary describes the gene products by molecular function, biological process and cellular component and its hierarchical structure allows both attribution and querying to be at different levels of specificity. In other words, one can focus on particular cellular functions or selected biological categories and analyze gene expression data of functionally clustered genes. Because GO annotation for the human genes could not be used directly to annotate gene lists generated by expression analysis, we resorted to the GenBank and SwissProt public GO gene association data compiled and made freely available by Compugen. By direct matching of the GenBank accession numbers or by referring to the GenBank and Swissprot accession numbers listed in the Unigene database we were able to assign ontology annotations to 8191 genes (near $70 \%$ of the total data set). Of these, 7180 were annotated for molecular function, 7246 for biological process and 6891 for cellular component, with 6580 genes having at least one annotation for each of the three main ontology terms. Next, meaningful biological categories were selected from the ontology hierarchy and annotated genes were clustered accordingly.

Table 1 summarizes the changes in the number of genes belonging to the different molecular function categories. Only gene transcripts detected in all the three independent experiments performed are reported. A molecular function category was attributed to 7141 genes out of 12600 represented in the HGU95A GeneChip array. The genes with definite function expressed in VD treated HL60 cells are 2965. Of these 2040 are not changed, 336 are increased, 417 are decreased. The proportion of genes whose expression is increased or decreased differs among the molecular function categories, indicating the important changes in gene expression underlying differentiation. Among the changes the most relevant to appear are those concerning the increased expression of genes encoding for ligand binding or carrier, receptors, cell surface antigens, defense immunity proteins, transporter and calcium binding and the decreased expression of genes belonging to DNA, RNA binding and chaperone categories.

Table 2 summarizes the changes occurring in VD treated cells concerning genes involved in different biological processes. An attribution to a biological process category has been possible for 7244 genes out of 12600 present in the HGU95A GeneChip array. The genes involved in biological process expressed in VD treated HL60 cells are 3026. Of these 2080 are not changed, 346 are increased and 412 are decreased. The proportion of genes whose 
expression changes in the different categories may be considered an indication of the biological processes involved in the differentiation transition. Interestingly, genes

Table 1 Changes in gene expression induced by VD treatment of HL60 cells. Molecular function categories

\begin{tabular}{lrrrrr}
\hline Function & ALL & \multicolumn{1}{c}{ P } & NC & \multicolumn{1}{c}{ I } & \multicolumn{1}{c}{ D } \\
\hline Calcium binding & 173 & 47 & 29 & $12^{*}$ & 4 \\
Cell adhesion molecule & 239 & 48 & 28 & 12 & 7 \\
Cell cycle regulator & 153 & 78 & 47 & 12 & 10 \\
Cell surface antigen & 124 & 24 & 14 & 9 & 2 \\
Chaperone & 123 & 70 & 45 & 4 & $20^{*}$ \\
Defense/immunity protein & 287 & 71 & 47 & $17^{*}$ & 5 \\
DNA binding & 480 & 261 & 185 & 13 & $50^{*}$ \\
Enzyme activator & 38 & 16 & 6 & 2 & 3 \\
Enzyme inhibitor & 176 & 55 & 35 & 10 & 6 \\
Enzyme & 2214 & 1015 & 691 & 103 & 165 \\
Ligand binding or carrier & 780 & 328 & 227 & $49^{*}$ & 27 \\
Ligand & 103 & 14 & 2 & 7 & 3 \\
Nucleic acid binding & 123 & 79 & 62 & 4 & 14 \\
Protein kinase & 337 & 139 & 98 & 11 & 16 \\
Protein phosphatase & 113 & 53 & 31 & 13 & 7 \\
Receptor & 166 & 15 & 5 & $8^{*}$ & 0 \\
RNA binding & 335 & 238 & 195 & 1 & $41^{*}$ \\
Signal transducer & 39 & 12 & 9 & 1 & 1 \\
Structural protein & 93 & 23 & 20 & 0 & 1 \\
Transcription factor & 844 & 342 & 241 & 41 & 33 \\
Transporter & 201 & 37 & 23 & 7 & 2 \\
Total & 7141 & 2965 & 2040 & 336 & 417 \\
\hline
\end{tabular}

*Significantly different with respect to the overall frequency of increased and decreased genes (test $\gamma^{2} \quad P<0.01$ ). ALL indicates the total number of genes present in the chip and categorized by molecular function. ' $P$ ' indicates the number of such genes expressed (called 'present' in all the experiments performed) in VD treated cells. 'NC', 'I', and 'D', indicate the number of genes respectively called 'not changed', 'increased', and 'decreased' in VD treated cells compared with the baseline (proliferating not treated cells) in all the experiments performed

Table 2 Changes in gene expression induced by VD treatment of HL60 cells. Biological function categories

\begin{tabular}{lrrrrr}
\hline Function & ALL & \multicolumn{1}{c}{ P } & NC & \multicolumn{1}{c}{ I } & D \\
\hline Apoptosis & 236 & 115 & 69 & $22^{*}$ & 12 \\
Cell communication & 497 & 104 & 63 & 18 & 12 \\
Cell cycle & 393 & 246 & 179 & 9 & $51^{*}$ \\
Cell growth and & 430 & 203 & 146 & 22 & 22 \\
$\quad$ maintenance & & & & & \\
Cell motility & 112 & 32 & 20 & 7 & 2 \\
Cell organization and & 371 & 182 & 127 & 8 & 29 \\
$\quad$ biogenesis & & & & & \\
Death & 23 & 4 & 1 & 2 & 2 \\
Developmental processes & 365 & 96 & 67 & 13 & 15 \\
Metabolism & 2650 & 1318 & 963 & 107 & 197 \\
Response to external & 464 & 159 & 90 & $38^{*}$ & 21 \\
$\quad$ stimulus & & & & & \\
Signal transduction & 1260 & 427 & 264 & $78^{*}$ & 35 \\
$\quad$ Stress response & 14 & 6 & 3 & 1 & 1 \\
$\quad$ Transport & 429 & 134 & 88 & 21 & 13 \\
Total & 7244 & 3026 & 2080 & 346 & 412 \\
\hline
\end{tabular}

*Significantly different with respect to the overall frequency of increased and decreased genes (test $\chi^{2} \quad P<0.01$ ). 'ALL' indicates the total number of genes present in the chip and categorized by biological process. ' $P$ ' indicates the number of such genes expressed (called 'present' in all the experiments performed) in VD treated cells. 'NC', 'l', and ' $D$ ', indicate the number of genes respectively called 'not changed', 'increased', and 'decreased' in VD treated cells compared with the baseline (proliferating not treated cells) in all the experiments performed involved in apoptosis, cell communication, cell motility, response to external stimuli, signal transduction and transport are increased. On the contrary, a decreased gene expression is observed for biological processes such as cell cycle, cell organization and biogenesis and metabolism.

Table 3 reports gene expression variations concerning cellular components. A total number of 6855 out of 12600 genes represented in the HGU95A GeneChip array encode for proteins localized in the different cellular components and 2850 are expressed in VD treated HL60 cells. Of these 2036 are not changed, 309 are increased and 409 are decreased, indicating that differentiation is characterized by a trend toward a reduced gene expression in the majority of cellular components, particularly in nucleus, nucleolus, nuclear membrane, mitochondrial and cytoplasm compartments. Increased gene expression occurs only in extracellular, integral plasma membrane proteins, plasma membrane and lysosomal components.

Figure 2 shows the distribution of groups of genes characterized by different molecular functions whose expression does not change in VD treated cells (about $90 \%$ ). The large majority of these genes is represented by enzymes (34\%) and nucleic acid binding factors, i.e RNA binding, DNA binding, nucleic acid binding and transcription factors (33\%). These genes most likely represent the core of this differentiation transition. Worthy of mention is that functional categories more closely related to monocyte functions such as defense/immunity, cell adhesion molecule, signal transducer, receptor and ligand are present in a very low proportion or absent.

Table 3 Changes in gene expression induced by VD treatment of HL60 cells. Cellular component categories

\begin{tabular}{lrrrrr}
\hline Function & ALL & P & NC & I & D \\
\hline Chromosome & 120 & 68 & 53 & 5 & 10 \\
Cytoplasm & 916 & 447 & 337 & 37 & 59 \\
Cytoplasmic vesicle & 57 & 23 & 18 & 2 & 2 \\
Cytoskeleton & 417 & 127 & 85 & 15 & 17 \\
Cytosol & 233 & 149 & 123 & 8 & 14 \\
Endoplasmic reticulum & 239 & 108 & 94 & 6 & 10 \\
Extracellular & 568 & 98 & 49 & $26^{*}$ & 10 \\
Golgi apparatus & 196 & 89 & 70 & 5 & 8 \\
Integral plasma membrane & 834 & 173 & 90 & $42^{*}$ & 17 \\
$\quad$ protein & & & & & \\
Lysome & 145 & 64 & 34 & $21^{*}$ & 6 \\
Membrane & 93 & 30 & 15 & 8 & 4 \\
Mitochondrial membrane & 192 & 125 & 99 & 8 & 18 \\
Mitochondrion & 305 & 176 & 122 & 13 & 39 \\
Nuclear membrane & 557 & 253 & 183 & 23 & 42 \\
Nucleus & 1475 & 718 & 529 & 59 & 127 \\
Nucleolus & 63 & 46 & 32 & 0 & $14^{*}$ \\
Peroxisome & 130 & 71 & 56 & 5 & 7 \\
Plasma membrane & 315 & 85 & 47 & $26^{*}$ & 5 \\
Total & 6855 & 2850 & 2036 & 309 & 409 \\
\hline
\end{tabular}

*Significantly different with respect to the overall frequency of increased and decreased genes (test $\chi^{2} \quad P<0.01$ ). ALL indicates the total number of genes present in the chip and categorized by cellular component. ' $P$ ' indicates the number of such genes expressed (called 'present' in all the experiments performed) in VD treated cells. 'NC', (l', and 'D', indicate the number of genes respectively called 'not changed', 'increased', and 'decreased' in VD treated cells compared with the baseline (proliferating not treated cells) in all the experiments performed 


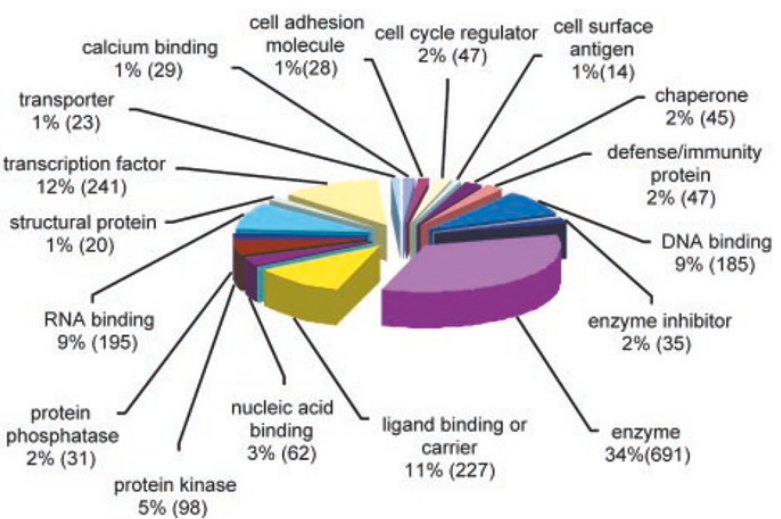

Figure 2 Proportion of categories of genes with different molecular function identified by Gene Ontology terms, whose expression does not change during HL60 differentiation process. Between parentheses the number of genes for each category is reported

To get a better definition of what is most significant in the changes of gene expression during the differentiation process, we have selected a group of genes whose abundance is significantly higher in VD treated HL60 cells (sort score higher than 10). Genes numberly 229 are included in this group and the representation of the different molecular function categories is shown in Figure 3. In this figure the number of genes in different categories is slightly different from that in Table 1, since the functional identification was performed by automated GO classification integrated with OMIM and Medline screening. It is evident that this representation is quite different from that reported in Figure 2 concerning the molecular function of the unchanged genes. In fact molecular function categories such as defense immunity, ligand, receptor, cell adhesion molecules and cell surface antigen, which are definitely associated with natural immunity, are among the most represented functional categories, whereas these genes are proportionally less represented among the functional categories of the unchanged genes. It is worth mentioning that the most decreased genes in VD treated HL60 cells (sort score less than 10) are included in the enzyme category (data not shown).

\section{VD induced and down-regulated gene expression}

As a consequence of VD treatment of HL60 cells, 115 genes, absent in untreated HL60 cells, are induced, but so far a definite function has been attributed only to 82 . Table 4 shows these genes subdivided by their molecular function. Sixty-one genes are completely down-regulated, and among them only 43 have a definite function (data not shown). It is necessary to point out that the sort score or fold change parameters should be considered only as an indication of the relative mRNA abundance since, at variance of increased, decreased and not changed mRNAs, induced and down regulated genes lack a proper term of comparison, because the fold change is calculated over the background noise and is an approximation. For this reason we have reported in Table 4 the average difference as an indication of mRNA abundance. Among the

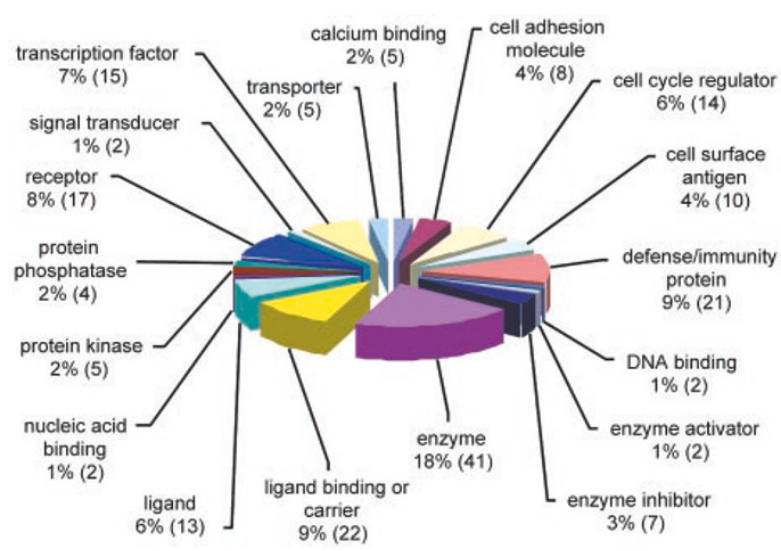

Figure 3 Proportion of categories of genes with different molecular function identified by gene ontology classification integrated with OMIM and Medline screening, whose expression is highly increased (sort score higher than 10) in $\mathrm{VD}$ treated cells. Between parentheses the number of genes for each category is reported

genes that are unexpectedly induced in VD treated cells and expressed at a high level are parvalbumin, able to regulate the release of calcium from the cell, and ninjurin, an adhesion molecule playing a role in tissues regeneration and function. Also worthy of attention are tenascin, an inhibitor of cell migration and ligand for several integrines, and PIG11, one of the p53 induced genes, able to activate an apoptotic pathway. Interestingly enough, this gene is expressed in spite of the deletion of p53 gene in HL60 cells. Another role is played by FALL39, expressed at high level and endowed with intense antibacterial activity. The expression of high levels of CKRX, a novel leukocyte chemokine receptor, is also of particular interest. It has also to be emphasized that we have observed in VD induced cells, the induction of the expression of genes so far considered typical of other types of blood cells: NCF2 and a neuthrophil collagenase as far as granulocytes are concerned and Adenosine A2 receptor as far as platelets are concerned. Among the down-regulated genes the most important decrease concerns the gene of the immunoglobulin lambda light chain and the BrCa1 proto-oncogene. As mentioned in Material and Methods, some of these differential expression pattern have been checked by quantitative real time PCR. The results (data not shown) completely confirm the DNA chip hybridization data.

\section{Comparison of the molecular phenotypes of VD treated HL60 cells and CD14+ peripheral blood monocytes}

To evaluate whether VD induced cells, obtained by the in vitro treatment of HL60 cells with $10^{-7} \mathrm{M}$ VD, are comparable with normal peripheral blood monocytes, we have examined the expression profile in two independent experiments using RNA extracted from CD14+ monocytes pooled from six normal donors and VD induced HL60 cells. Figure 4 summarizes the results of the comparison between the data obtained from the replicates of the two cellular contexts.

Genes are expressed in VD treated cells numbered 4902, whereas the genes expressed in CD14+ cells are 
Table 4 Genes specifically induced by VD treatment of HL60 cells

\begin{tabular}{|c|c|c|}
\hline & Avg Diff & GenBank \\
\hline \multicolumn{3}{|l|}{ Calcium binding } \\
\hline Calcium-binding protein in amniotic fluid 1 & 2640.33 & D83664 \\
\hline Parvalbumin & 1535.8 & X63578 \\
\hline \multicolumn{3}{|l|}{ Cell adhesion molecule } \\
\hline Major group rhinovirus receptor (HRV) & 3161.8 & Y00093 \\
\hline Osteopontin & 2984.47 & U17760 \\
\hline Leukocyte adhesion glycoprotein p150 & 3161.8 & J03925 \\
\hline Lamin S B3 & 2434.8 & U91512 \\
\hline $\begin{array}{l}\text { Mac- } 1 \text { gene encoding complement } \\
\text { receptor type } 3\end{array}$ & 14505 & J03925 \\
\hline Adhesion molecule ninjurin & 18300.1 & U91512 \\
\hline Laminin $\mathrm{B} 2$ chain & 1854.17 & M55210 \\
\hline Osteopontin mRNA & 1470.97 & J04765 \\
\hline Tenascin-C, 7650 bp & 1312.57 & X78565 \\
\hline \multicolumn{3}{|l|}{ Cell cycle regulator } \\
\hline Breast cancer suppressor candidate 1 & 1111 & AF002672 \\
\hline $\mathrm{Bcl}-2$ related & 3995.43 & U27467 \\
\hline Pig11 & 1750.03 & AF010315 \\
\hline Semaphorin E & 620.5 & AB000220 \\
\hline \multicolumn{3}{|l|}{ Cell surface antigen } \\
\hline Leukocyte antigen CD37 & 2321.63 & X14046 \\
\hline NMB & 2328.97 & L09230 \\
\hline CD9 antigen & 586.53 & M27492 \\
\hline EX-1=radiation-inducible immediate-early & 1849.87 & S81914 \\
\hline Putatively prenylated protein & 1004.2 & Y13374 \\
\hline \multicolumn{3}{|l|}{ Defense/immunity protein } \\
\hline C-C chemokine receptor type 1 (C-C CKR-1) & 2690.83 & L09230 \\
\hline Interleukin 1 receptor & 1627.97 & M27492 \\
\hline IgG Fc receptor hFcRn & 8165.17 & U12255 \\
\hline Fc alpha receptor & 4268.9 & U43774 \\
\hline FALL-39 peptide antibiotic & 30759.7 & Z38026 \\
\hline IgG Fc receptor $h F c R n$ & 8165.17 & U12255 \\
\hline IL-4-R mRNA for the interleukin 4 receptor & 5270.9 & $\times 52425$ \\
\hline V7 mRNA for leukocyte surface protein & 2269 & Z33642 \\
\hline Complement receptor type 1 & 1997.3 & $\mathrm{~L} 17418$ \\
\hline Interleukin-10 receptor & 1486.97 & U00672 \\
\hline Complement receptor type 1 & 1298.03 & Y00816 \\
\hline Chemokine receptor $\mathrm{X}$ (CKRX) & 4950.4 & AJ242015 \\
\hline \multicolumn{3}{|l|}{ DNA binding } \\
\hline $\begin{array}{l}\text { v-maf musculoaponeurotic fibrosarcoma } \\
\text { oncogene }\end{array}$ & 1083.07 & AL031228 \\
\hline Early growth response 2 protein & 978.1 & X92720 \\
\hline \multicolumn{3}{|l|}{ Enzyme } \\
\hline Guanylate binding protein isoform II & 1299.77 & X90858 \\
\hline Ecto-ATP diphosphohydrolase & 1608.37 & M62840 \\
\hline eMDC II protein & 687.2 & M55067 \\
\hline N-sulphoglucasamine sulphohydrolase & 6004.33 & M32011 \\
\hline beta3-Galactosyltransferase & 1367 & L13286 \\
\hline Phosphoenolpyruvate carboxykinase & 1288.53 & X92720 \\
\hline Carboxylesterase & 18709.8 & L07765 \\
\hline Uridine phosphorylase & 479.27 & X90858 \\
\hline Acyloxyacyl hydrolase & 1198.47 & M62840 \\
\hline $\begin{array}{l}\text { 47-kD autosomal chronic granulomatous } \\
\text { disease protein }\end{array}$ & 18301.6 & AL049699 \\
\hline Neutrophil oxidase factor (p67-phox) & 13183.3 & M32011 \\
\hline $\begin{array}{l}\text { Mitochondrial 1,25-dihydroxyvitamin D3 } \\
\text { 24-hydroxylase }\end{array}$ & 17157.9 & M36821 \\
\hline Collagenase & 7168.2 & J05556 \\
\hline Skeletal muscle-specific calpain & 2458.7 & X85030 \\
\hline Arginase & 1582.43 & M14502 \\
\hline Cytochrome $\mathrm{P} 450$ reductase & 2214.63 & S90469 \\
\hline Malic enzyme 1 malate oxidoreductase & 562.9 & AL049699 \\
\hline Glutamine cyclotransferase & 12634.9 & X71125 \\
\hline \multicolumn{3}{|l|}{ Ligand } \\
\hline Cytokine (GRO-gamma) & 1399.23 & U52112 \\
\hline (Act-2) mRNA & 25606.3 & AB006780 \\
\hline Haptoglobin alpha(2FS)-beta precursor & 5596.87 & X00442 \\
\hline Cytokine Gro beta & 898.4 & M36820 \\
\hline Endothelin-1 (EDN1) gene & 6580.63 & J05008 \\
\hline
\end{tabular}

Table 4 continued

\begin{tabular}{|c|c|c|}
\hline & Avg Diff & GenBank \\
\hline Transforming growth factor-beta & 4913.73 & M60315 \\
\hline Chemokine exodus- 1 & 2411.8 & U64197 \\
\hline \multicolumn{3}{|l|}{ Ligand binding or carrier } \\
\hline Renin-binding protein & 2204.17 & U52112 \\
\hline Galectin-3 & 1226.23 & AF027826 \\
\hline Necrosis factor-inducible & 5014.5 & M31165 \\
\hline Fibronectin & 3832.83 & X02761 \\
\hline $\begin{array}{l}\text { TTF small G protein } \\
\text { Nucleic acid binding }\end{array}$ & 1382.87 & Z35227 \\
\hline \multicolumn{3}{|l|}{ Nucleic acid binding } \\
\hline $\begin{array}{l}\text { Oncogene Tls/Chop fusion Activated } \\
\text { Protein kinase }\end{array}$ & 3307.03 & S62138 \\
\hline Putative serine/threonine protein kinase & 8323.3 & Y10032 \\
\hline Ndr protein kinase & 916.63 & Z35102 \\
\hline \multicolumn{3}{|l|}{ Protein phosphatase } \\
\hline MAP kinase phosphatase (MKP-2) & 1107.77 & AF063002 \\
\hline \multicolumn{2}{|l|}{ Receptor } & L08488 \\
\hline Putative seven pass transmembrane protein & 675.6 & AF027826 \\
\hline A2 receptor [human hippocampal] & 4345.83 & S46950 \\
\hline G protein-coupled receptor kinase & 4417.47 & L15388 \\
\hline Heparin-binding EGF-like growth factor & 3799.47 & M60278 \\
\hline LDL-receptor related protein & 4111.57 & X13916 \\
\hline Lectin-type oxidized LDL receptor & 2125.93 & AF079167 \\
\hline \multicolumn{3}{|l|}{ Signal transducer } \\
\hline Helix-loop-helix basic phosphoprotein & 2063.37 & AL050262 \\
\hline \multicolumn{3}{|l|}{ Structural protein } \\
\hline Fibromodulin mRNA & 3832.83 & U05291 \\
\hline \multicolumn{3}{|l|}{ Transcription factor } \\
\hline Activating transcription factor 3 & 376.4 & Al632589 \\
\hline LIM protein SLIMMER & 2506.57 & AL022318 \\
\hline c-jun proto oncogene & 2916.9 & AF071504 \\
\hline Orphan nuclear receptor & 4030.5 & AF009425 \\
\hline Homeodomain protein & 1348.43 & AF038187 \\
\hline $\begin{array}{l}\text { Peroxisome proliferator activated receptor } \\
\text { gamma }\end{array}$ & 1299.23 & AF052288 \\
\hline Forkhead protein FREAC-1 & 1107.7 & AB018259 \\
\hline ERF-1 mRNA 3 end & 2403.2 & X79067 \\
\hline I-Rel mRNA & 1016.63 & M83221 \\
\hline Epstein-Barr virus-induced protein & 966.83 & U19261 \\
\hline \multicolumn{3}{|l|}{ Transporter } \\
\hline AQP9 mRNA for aquaporin 9 & 11409.2 & AB008775 \\
\hline \multicolumn{3}{|l|}{ Molecular function unknown.F } \\
\hline KIAA0394 & 1909.7 & AB007854 \\
\hline cDNA DKFZp547I0610 & 2206.97 & AL050262 \\
\hline KIAA0119 gene & 1196.53 & D17793 \\
\hline $\begin{array}{l}\text { dJ377H14.9 major histocompatibility } \\
\text { complex }\end{array}$ & 2578.6 & AL022723 \\
\hline KIAA0500 & 1196.53 & AB007969 \\
\hline wb10h03.x1 Homo sapiens cDNA & 1711.4 & Al632589 \\
\hline bK150C2.2 (Phorbolin 3) & 1956.97 & AL022318 \\
\hline Syntaxin 11 mRNA & 1053.8 & AF071504 \\
\hline Clone 22 & 897.03 & AF009425 \\
\hline Clone 23714 & 2313.13 & AF038187 \\
\hline Huntingtin interacting protein 1 & 579.5 & AF052288 \\
\hline KIAA0716 protein & 951.77 & AB018259 \\
\hline G0S2 protein & 38727.5 & M69199 \\
\hline Clone 24775 & 18780 & AF052169 \\
\hline zx53d03.r1 & 10623.5 & AA203487 \\
\hline zb11b10.s1 Homo sapiens cDNA, 3 end & 9902.33 & N90866 \\
\hline $51 a 9$ & 5003.57 & W28743 \\
\hline KIAA0206 & 1932.1 & D86961 \\
\hline PAC clone DJ0751H13 & 3812.87 & AC004877 \\
\hline DKFZp586E1621 & 1603.43 & AL080235 \\
\hline dJ434014.3.3 & 1976.47 & AL022398 \\
\hline Clone 23765 & 1366.93 & AF052097 \\
\hline KIAA0823 & 784.47 & AB020630 \\
\hline 31 a10 Human & 2059.23 & W27419 \\
\hline KIAA0513 & 3604.1 & AB011085 \\
\hline HSZ78315 & 1089.17 & Z78315 \\
\hline Disintegrin-protease & 542.03 & Y13323 \\
\hline
\end{tabular}


4687. The genes whose expression levels are substantially the same are 2879, whereas 384 are more expressed in CD14+ cells and 643 in VD treated cells. Specifically expressed genes, 219 in CD14+ monocytes whereas 224 in VD treated HL60 cells. On the other side, as previously explained, there is a fraction of very low abundant sequences, 562 in CD14+ cells and 772 in VD treated cells whose abundance is near the detection threshold and that are not further considered in this study.

The comparison between the CD14+ and VD treated HL60 cells specific genes, based on the molecular functions as reported in Figure 5, shows that a remarkable difference is observed mainly in the defense/immunity category since CD14+ monocytes express specifically several class II MHC molecules as reported in Table 5. Genes that are specifically expressed in VD treated HL60 cells are not separately reported since the majority are included in VD induced genes reported in Table 4 . In this

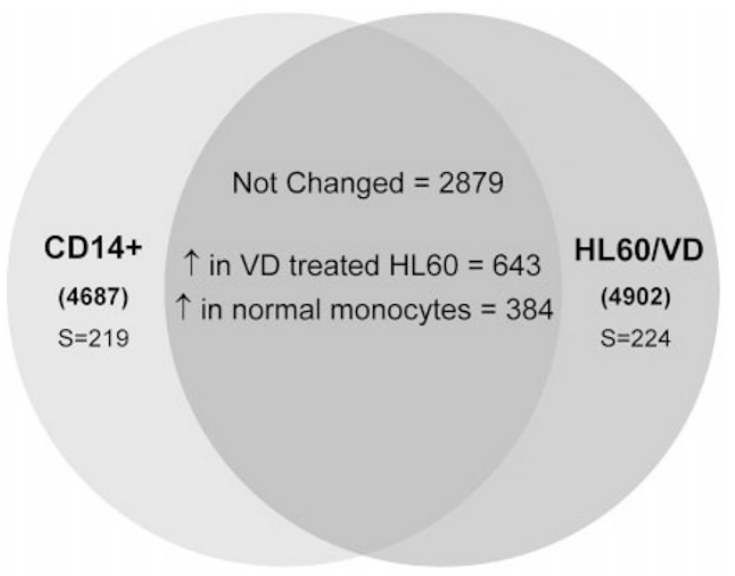

Figure 4 The diagram shows the overall number of genes present in VD treated HL60 cells and CD14+ peripheral monocytes subdivided according to their relative abundance. The term 'specific' (S) indicates those genes whose expression was present only in one type of cell and absent in the other type

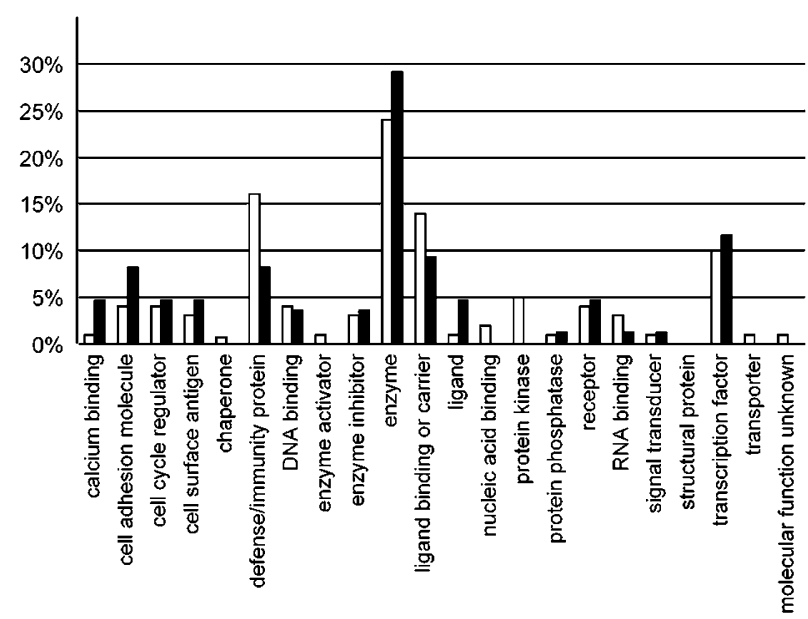

Figure 5 The histogram shows comparatively the distribution of specifically expressed genes in CD14+ (white bars) and VD treated HL60 cells (black bars) classified according to their molecular function
Table the genes involved in mechanisms of natural immunity specific of VD treated cells are also listed.

\section{Effect of VD on the expression of defense/ immunity molecules in CD14+ monocytes activated by serum treatment}

The comparison of the set of genes specifically expressed in VD treated cells and CD14+ monocytes clearly indicates that

Table 5 Genes related to natural immunity specifically expressed in CD14+ monocytes

\begin{tabular}{lcc}
\hline & Avg Diff & GenBank \\
\hline Defense/immunity protein & & \\
MHC, class II, DP beta 1 & 15560.4 & M83664 \\
MHC, class II, DQ beta 1 & 8616 & M60028 \\
MHC, class II HLA-DQ-(DR7 DQw2) & 9927.6 & M81141 \\
MHC, class II, DQ beta 1 & 6603.6 & M16276 \\
MHC, class II, DR beta 4 & 3283.2 & M16942 \\
MHC, class II, DQ alpha & 632.4 & AA868382 \\
MHC, class II, DR alpha & 52743.6 & J00194 \\
MHC, class II, DM alpha & 13075.2 & X62744 \\
MHC, class II, DP alpha & 41592 & X00457 \\
MHC, class II, DR beta 1 & 17869.2 & M32578 \\
fms-related tyrosine kinase 3 & 378 & U02687 \\
Heat shock 70 kD protein 1B & 2576.4 & M59830 \\
Triple functional domain (PTPRF & 859.2 & AF091395
\end{tabular}

interacting)

la-associated invariant gamma-chain gene $41906.4 \quad$ M13560

Coagulation factor XIII, A1 polypeptide $10250.4 \quad$ M14539

Immunoglobulin-like transcript 3 protein $\quad 3634.8$ AF072099

variant 1 gene

Myxovirus (influenza) resistance 1, $1776 \quad$ M33882

homolog of murine

Fc fragment of IgG, low affinity IIlb, receptor for (CD16)

Thymocyte antigen CD1c

Toll-like receptor 5

Coagulation factor $\mathrm{V}$

T-cell receptor active beta-chain

Sialic acid binding lg-like lectin 7

Leucocyte immunoglobulin-like receptor-6a (LIR-6)

Immunoglobulin lambda locus

Fc fragment of $\operatorname{lgE}$, high affinity I, receptor for; alpha

1.6 Kb mRNA for 2-5A synthetase induced by interferon

Ligand

TNF-related apoptosis inducing ligand TRAIL

Interleukin 15 (IL15)

Interleukin 15 precursor (IL-15)

Receptor

Macrophage receptor MARCO

Leucocyte immunoglobulin-like receptor-6b (LIR-6)

Leucocyte immunoglobulin-like receptor-4 (LIR-4)

Interleukin-10 receptor

Chemokine (C-X3-C) receptor 1

ccr2b (ccr2)

Chemokine ( $\mathrm{C}-\mathrm{C}$ motif) receptor 2

927.6

$\mathrm{X} 16863$

815.6

1124.4

958.8

1716

1340.4

570

M28827

AF051151

M16967

M12886

AJ007395

AF025530

612

598.8

M18645

1370.4

X06948

Transcription factor

Transcription factor 3 (E2A immunoglobulin enhancer binding)

Interferon regulatory factor 4

Interferon regulatory factor 5

3122.4

U37518

301.2

U14407

811.2 AF031167

1531.2 AF035819

1940.4 AF025529

1030.8

AF025527

13554

5482.8

U00672

U95626

$1005.6 \quad$ U03905

$1009.2 \quad$ M65214

$817.2 \quad$ U52682

$7480.8 \quad$ U51127 
the latter cells express higher numbers of mRNAs involved in the mechanisms of natural immunity. To obtain further informations about the effects of VD on the expression of defense/immunity molecules, we have analyzed the gene expression profiles of CD14+ monocytes activated by serum either in the absence or in the presence of pharmacological doses of VD. Figure 6 shows the comparison of the overall number of genes expressed in untreated, serum stimulated and serum stimulated plus VD monocytes. In this comparison the attention has been focused on about 180 genes categorized as defense/immunity. This category has been divided in 16 sub-categories based on both molecular function and biological process as shown in Figure 7. It must be pointed out that several genes whose expression is increased or induced by serum treatment of CD14+ monocytes are the same as those described as increased in dendritic cells (data not shown). Among the defense/immunity sub-categories, by far the most significant changes in the expression pattern has been observed in class II MHC genes. As shown in Figure 8A, that regards the comparison of class II MHC genes expression changes, the serum treatment of CD14+ monocytes induces an increased expression of all these genes, whereas the simultaneous treatment with VD not only inhibits this increase but leads to expression levels lower than that observed in untreated monocytes. It is important to call attention to the fact that DRB5 and DMB genes, expressed, at low level, in proliferating HL-60 cells, are completely downregulated by VD treatment. Since VD may interfere with the translational activity, we checked the level of DR protein by flow cytometry to verify the correspondence with the level of

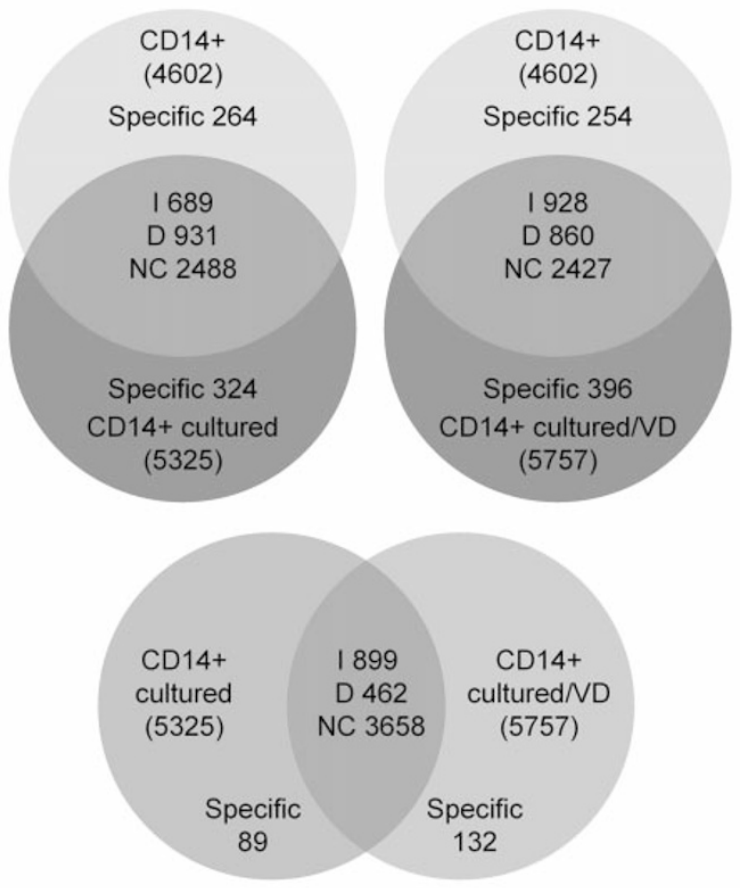

Figure 6 The diagrams show the comparison of the overall number of genes expressed in untreated (CD14+), serum stimulated (CD14+ cultured) and serum stimulated plus VD (CD14+ cultured/VD) monocytes subdivided according to their relative abundance. The term 'specific' indicates those genes whose expression was present only in one type of cell and absent in the other type
mRNA. Our results, reported in Figure 8B, clearly show a very good correlation between DR mRNA and protein levels.

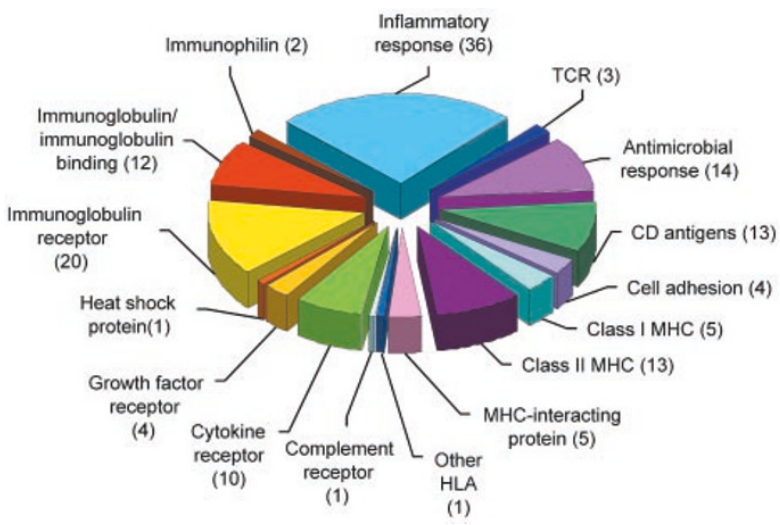

Figure 7 The diagram shows the 16 subcategories based on both molecular function and biological process that characterize the defense/immunity main category. Between parentheses the number of genes for each category is reported

A
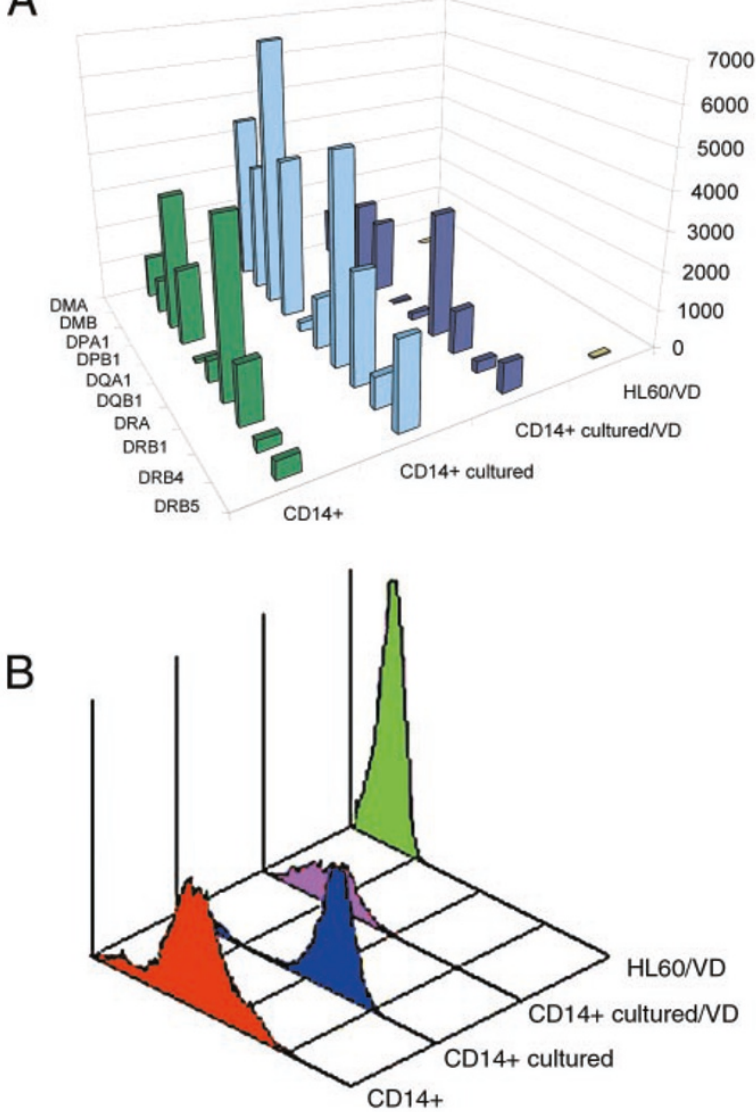

Figure 8 (A) The histogram shows the abundance (expressed as average difference) of class II MHC molecules transcripts in untreated (CD14+), serum stimulated (CD14+ cultured), serum stimulated plus VD (CD14+ cultured/VD) monocytes and in VD treated HL60 cells. (B) Flow cytometric analysis of DR expression in the same samples. Fluorescence data are expressed as mean channel fluorescence (MCF) and as percentage (\%) of positive cells: CD14+ (MCF=8.9, \%=91.8); CD14+ cultured $(\mathrm{MCF}=31, \%=91.2) ; \mathrm{CD} 14+$ cultured/VD $(\mathrm{MCF}=3, \%=57) ; \mathrm{HL} 60$ VD $(\mathrm{MCF}=1.9, \%=0.5)$ 


\section{Discussion}

The purpose of our study was to obtain indications about the biological behavior changes, indicated by gene expression profiles, underlying the differentiation transition between myeloblasts and monocytes, and to compare the molecular phenotypes of spontaneously and pharmacologically differentiated cells.

The results have been obtained by evaluation and comparison of three independent experiments. This criterion, largely accepted by many authors, has the advantage of allowing the selection of those genes that are constantly expressed, avoiding the problem represented by the biological variability characterizing each experiment. It is important to point out that we have taken into consideration in this analysis only those genes whose expression behavior, increased, decreased, unchanged or specific, was uniform in all the experiments.

The use of Gene Ontology hierarchical vocabulary allowed us to cluster genes according to their functional properties, thus enlightening biological processes or pathways that are affected by the VD induced monocytic differentiation. Even though the ontology-based system of gene clustering can provide more reliable results with respect to other categorization methods (e.g. the use of SwissProt keywords), it still suffers from some limitations. These are mainly due to the incompleteness of the GO vocabulary, the scarcity of functional annotations for human genes, and the subjectivity of the category selection process. While the first two limitations will be overcome in the next years by the improvement of annotation accuracy, the third one would require the development of dedicated computer methods. A computer program aimed at selecting on a statistical basis ontology categories for which gene expression is significantly varied in microarrays experiments is currently under study in our laboratory. In our experiments we have used the active form of VD to induce a phenotypic conversion to monocytes of leukemic cells of the HL60 cell line. This differentiating activity, like that of other agents such as ATRA or Butyric acid derivates, is actually under investigation since it represents a possibility to overcome the differentiation block characterizing AML blast cells. ${ }^{20,21}$ Hence our interest to explore, by DNA microarrays, the changes of genome expression underlying the differentiation transition from myeloblasts to monocytes. A further goal of our study was to evaluate whether the monocytic conversion of HL60 cells, induced by VD, leads to a cellular element that corresponds to the molecular phenotype of CD14+ peripheral blood monocyte. First of all it is important to point out that only $10 \%$ of genes are differentially expressed in this differentiation transition. In spite of the fact that we have taken into account only those genes constantly expressed in three different experiments, a significant proportion of the transcriptome has a variability in the expression behavior, and for this reason has not been considered in our analysis. However, the sensitive DNA arrays technology allows us to identify a subset of genes that definitely varies in their expression during the differentiation. Very useful to the characteriza- tion of the differentiation process is the subdivision of differentially expressed genes based on molecular function, biological process and cellular component according to Gene Ontology.

As already shown in the Results, in VD induced HL60 cells there is a relative increase of gene expression in the following molecular functional categories: calcium binding, transporter, defense/immunity protein, cell surface antigen, receptor and ligand binding or carrier. On the contrary, a remarkable decrease is observed for nucleic acid binding, DNA binding, RNA binding, chaperone and enzyme categories. These data clearly suggest that the gene expression in VD treated cells is oriented toward exteriorization, transport, protein secretion and defense. A confirmation comes when we consider genes involved in biological processes. In fact, genes related to response to external stimuli, transport, cell motility, cell communication, signal transduction and apoptosis have an increased expression. In contrast, the expression of genes involved in cellular metabolism is drastically decreased as well as that of genes involved in cell cycle, cell organization and biogenesis. The clearest indication of the expression behavior characterizing the phenotypic conversion to monocytes is obtained when we analyze the changes in the expression of genes grouped in cellular components. In fact, the expression of genes encoding for most cell components is reduced, with the exceptions of those coding for extracellular, plasma membrane, integral plasma membrane and lysosomes. The reduction is particularly evident among genes related to nucleus, nuclear membrane, mithocondria and mainly nucleolus.

As a whole, these results indicate that the monocyte phenotypic conversion of HL60 cells treated with VD implicates a differential expression of a relatively small number of mRNAs and the induction or down-regulation of an even smaller number of genes. The result of these changes in gene expression profile leads to a different biological behavior of monocytes, since an increased expression of mRNAs involved in surface and external activities occurs while these cells progressively loose the ability to increase or even maintain the expression of mRNAs involved in internal structures. In support of this conclusion comes the observation that several of the newly induced genes in VD treated cells, belong to adhesion molecule, defense/immunity, enzyme, ligand, receptor and surface molecule categories.

We have chosen to give a general picture of the differentially expressed genes rather than to analyze these changes in detail and only genes related to natural immunity will be discussed in some details.

The comparison between the molecular phenotypes of VD induced HL60 cells and CD14+ peripheral blood monocytes has been performed to establish whether the action of VD induces a phenotypic conversion similar to normal monocytes. The analysis of the gene expression profile shows that a large proportion of the expressed genes is common to the two cellular contexts, even though a significant number of genes has a considerable variability in their abundance. It is important to note that in the limited set of genes specifically expressed in VD cells but not in 
CD14+ monocytes, more than $50 \%$ are common to the genes specifically induced during the differentiation program activated by VD. This leads to the believe that this genetic program is at least partially divergent from that underlying physiological monocytopoiesis. In fact when we consider the molecular function of cell type specific genes we can see that CD14+ monocytes express a number of class II MHC molecules, whereas VD treatment downregulates the class II MHC molecules expressed, even if at low level, in HL-60 cells.

Furthermore, we have compared our results with that of other authors who have studied by DNA microarrays the expression profile of CD14+ monocytes activated to dendritic cells. ${ }^{17,18,22}$ This comparison clearly indicates that VD treated cells do not express gene involved in macrophagic activation.

To obtain further evidence about the ability of VD to interfere with the class II MHC gene expression or the expression of other genes of the defense/immunity category, we have compared the gene expression profile of CD14+ monocytes activated by serum in the presence or absence of VD. Our results confirm that VD inhibits the increased expression of class II MHC genes due to serum stimulation without affecting significantly the expression of other defense/immunity genes including class I MHC or immunoglobulin receptors genes.

Our results are in keeping with that of Berer et al. ${ }^{23}$ who observed the inhibition of dendritic cell differentiation from monocytes by blocking induction or up-regulation of characteristic DC associated molecules such as class II MHC molecules. These authors have also observed a marked suppression of T cell stimulatory function of DCs by VD. Tokuda and Levy ${ }^{24}$ have observed a strong inhibitory effect of VD on DR class II MHC antigen expression in cells exhibiting high phagocytic activity. They, therefore, speculate that VD could induce immature monocytes to differentiate into executive professional phagocytes at the potential expenses of immune antigen presenting cell function. However previous studies in our laboratory have shown that VD at physiological levels promotes a differentiation of CD34+ progenitors characterized by the induction of all the mono-macrophagic immunophenotypic and morphological markers. ${ }^{25}$ On the basis of these results we feel justified to speculate that when VD is physiologically involved in monocytic commitment of myeloid progenitors, the genetic program leads to a molecular phenotype different from that of CD14+ monocytes, particularly when the expression of class II MHC molecules is concerned.

\section{Materials and Methods}

\section{Cell populations}

HL60 cell line ${ }^{26}$ was obtained from ATCC and cultured, as already described $^{27}$, in RPMI 1640 medium (Life Technologies Inc., Gaithersburg, MD, USA), supplemented with $10 \%$ fetal bovine serum (FBS) (Life Technologies Inc., Gaithersburg, MD, USA) and 2 mM LGlutamine (Life Technologies Inc). Differentiation was induced by treatment with $10^{-7} \mathrm{M}$ VD (Hoffman-Laroche, Basel, Switzerland) for 5 days. Differentiation was monitored by May Grümwald Giemsa (MGG) staining and by immunophenotypic analysis with phycoerythrin-conjugated mouse anti-human CD11b and CD14 monoclonal antibodies (Becton Dickinson Immuno-cytochemistry Systems, San Jose, CA, USA), performed by flow cytometry as described below. Upon informed consent, six normal blood samples were obtained from three male and three female donors. From $100 \mathrm{ml}$ of peripheral blood mononuclear cells (PBMC) were isolated immediately by using a Ficoll-Hypaque gradient. CD14 positive cells were than purified from PBMC using an anti-CD14 antibody conjugated to magnetic beads (Miltenyi Biotec, Auburn, CA, USA) over a magnetic column as described by the manufacturer protocol (Miltenyi Biotec, Auburn, CA, USA). The purity of CD14+ selected cells was determined by flow cytometry and MGG staining. All samples were $>95 \%$ pure. One-third of CD14 positive cells were immediately lysed in Guanidinium isothiocyanate solution $(3.75 \mathrm{M}$ guanidinium isothiocyanate, $24.5 \mathrm{mM} \mathrm{Na}$ Cytrate, $0.48 \%$ Sarkosyl, $90 \mathrm{mM} \beta$-mercaptoethanol), one-third were cultured in IMDM supplemented with 20\%FBS, $10 \%$ filtered homolog serum and $2 \mathrm{mM}$ L-Glutamine for 3 days and the last third, cultured in the same conditions, were treated with $10^{-7} \mathrm{M}$ VD for 3 days. Cultured VD treated and untreated CD14 positive cells were then lysed in Guanidinium isothiocyanate.

\section{Flow cytometry}

A phycoerythrin-conjugated mouse anti-human CD11b and CD14 monoclonal antibodies (Becton Dickinson Immuno-cytochemistry Systems) were used for differentiation monitoring and a mouse monoclonal anti-human HLA II antigen FITC conjugated (Euro Clone, Devon, UK) was used for DR expression evaluation. Samples were analyzed with Epics XL flow cytometer (Coulter Electronics Inc., Hialeah, FL, USA) equipped with a single 488-nm argon laser and XL2 software (Coulter Electronics Inc.)

\section{RNA extraction}

Total cellular RNA was isolated in three independent experiments from $10^{7}$ proliferating and VD treated HL60 cells using the RNeasy RNA isolation system (Qiagen, Valencia, CA, USA) and following manufacturer's recommendations. Total cellular RNA was isolated from CD14+ cells using a modification of the guanidinium-cesium chloride centrifugation technique as already described. ${ }^{28}$ RNA was assessed by formaldehyde agarose gel electrophoresis, and quantitated by UV absorbance.

\section{Biotin-labeled cRNA transcription and gene-chip hybridization}

For biotin-labeled target synthesis, reactions were performed using standard protocols supplied by the manufacturer (Affymetrix, Santa Clara, CA, USA). Briefly, $5 \mu \mathrm{g}$ of the RNA was converted into doublestranded cDNA by reverse transcription using a cDNA synthesis kit (SuperScript Choice System, Invitrogen Ltd, Paisley, UK) using protocols supplied by the manufacturer with a T7-(dT) 24 primer (MWGBiotech, Ebesberg Germany). After second-strand synthesis, labeled cRNA was generated from the cDNA sample by an in vitro transcription reaction supplemented with biotin-11-CTP and biotin-16-UTP (Enzo, Farmingdale, NY, USA) as described by the manufacturer protocol. The labeled cRNA was purified by using RNeasy spin columns (Qiagen, Valencia, CA, USA). The concentration of biotin-labeled CRNA was determined by UV absorbance. In all cases, $15 \mu \mathrm{g}$ of each biotinylated cRNA preparation was fragmented, assessed by gel 
electrophoresis, and placed in a hybridization cocktail containing four biotinylated hybridization controls (BioB, BioC, BioD, and $\mathrm{Crex}$ ) as recommended by the Affymetrix protocol. Samples were hybridized to an identical lot of Affymetrix HGU95A GeneChip arrays for $16 \mathrm{~h}$. GeneChips were washed and stained using the instrument's standard Eukaryotic GE WS2 protocol, using antibody-mediated signal amplification.

\section{Data analysis}

Six HGU95A GeneChip arrays were hybridized from three independent differentiation induction of HL60 cells. Two HGU95A GeneChip arrays were hybridized from two independent pools of RNAs extracted from CD14+ peripheral blood monocytes as described above. The images from the scanned chips were processed using Affymetrix Microarray Analysis Suite 4.0 (MAS 4.0). The expression level of a single mRNA (average difference) was determined, using the MAS 4.0 absolute analysis algorithm, as the average fluorescence intensity among the intensities obtained by 16 to 20 paired (perfectly matched and single nucleotidemismatched) probes consisting of 25-base oligonucleotides. If the intensities of mismatched probes are very high, gene expression is judged to be 'absent' by the absolute call decision matrix even if a high average fluorescence is obtained. The MAS 4.0 comparison analysis algorithm was used in order to compare gene expression levels between two samples. The analysis employs normalization and scaling techniques to minimize differences in overall signal intensities between the arrays. The data were then analyzed using comparison algorithms to derive a different call which indicates whether a transcript is increased or decreased or exhibits no change in expression level. In addition a fold change calculation was carried out to indicate the relative change of each transcript represented on probe array. In order to better identify significant changes between transcripts we also used an other value calculated by comparison analysis algorithms: the sort score that is a ranking based on the fold change and the average difference change (the higher fold change and the average difference change, the higher sort score). The MAS4.0 generated absolute and comparison analysis data were then uploaded onto Affymetrix Micro DB database and processed with Affymetrix Data Mining Tool (DMT) 2.0 software. DMT 2.0 was used to query data from the three independent data sets and to sort genes that were called 'present' or 'absent' by absolute analysis and that were called 'increased' or 'decreased' or 'not changed' by comparison analysis in three out of three experiments. DMT was also used to calculate averages and standard deviations of average differences, average difference changes, fold change and sort scores of the independent data sets. The gene lists created by DMT software were transferred to Microsoft Excel and linked to Internet genome databases (e.g., GenBank, Swiss Prot, OMIM, and GeneCard).

\section{Functional clustering}

The Compugen flat file release 0.4.1 of Gene Ontology association data (http://www.cgen.com) was used to assign GO identifier to individual genes of the U95A data set. All the Compugen SwissProt and GenBank gene associations were inferred with predictive method and hence coded as IEA (inferred from electronic annotation). A few genes could be annotated by direct comparison of HGU95A data set and Compugen GenBank accession number. In most cases we used the Unigene accession number (that was available for 10567 probe sets) to retrieve a list of Genbank and SwissProt accession numbers for each HGU95A gene to be matched with the Compugen association data. All these comparisons were performed with custom Perl routines on a Unix workstation. The Gene Ontology database (http:// www.geneontology.org) was used to cluster annotated U95A genes for functional categories. Gene categories were chosen by browsing the three main terms of the ontology vocabulary: molecular function, biological process and cellular component. The database was remotely queried with a dedicated Perl program based on the Handle module of the GO Application Programming Interface (http:// www.fruitfly.org/annot/go). The selected categories were thus assigned to each annotated gene by parsing all parental terms of individual ontology identifiers. In many cases, single genes were associated with multiple identifiers for each main GO term. To avoid ambiguities, categories were sorted by relevance and a single attribution was made for each main ontology term. The ontologyannotated set of HGU95A genes may be downloaded from the site: http://biochimica.unipr.it/U95AGO.

\section{Real-Time Quantitative RT - PCR}

First strand of CDNA were synthesized starting from $1 \mu \mathrm{g}$ of total RNA extracted from HL60 and VD treated HL60 cells using the Super Choiche System for cDNA Synthesis (Invitrogen Ltd Paisley, UK) in presence of $100 \mathrm{pmol}$ oligo (dT)18 primer (Ambion Inc. Austin TX USA) and $200 \mathrm{U}$ SuperScript II. Fifty $\mu \mathrm{l}$ of $\mathrm{RT}-\mathrm{PCR}$ reaction (Quantitect SYBR Green PCR Kit Qiagen) volume included $1 \mu \mathrm{l}$ of first-strand CDNA reaction, 1X Quantitect SYBR Green PCR Master Mix, and $300 \mathrm{nM}$ specific primers. ICycler iQ ${ }^{\circledR}$ (Biorad Laboratories Hercules, CA, USA) was used to measure the relative abundance of mRNAs. RT-PCR cycle parameters were $95^{\circ} \mathrm{C} 15^{\prime \prime}$ followed by 40 cycles at $94^{\circ} \mathrm{C} 20^{\prime \prime}$ and $1^{\prime}$ at $60^{\circ} \mathrm{C}$. The primers used in the study were designed using Primer 3 software (http://www-genome.wi.mit.edu/cgibin/primer/primer3_www.cgi/) and synthesized by MWG-Biotech, (Ebesberg Germany). For osteopontin the forward and the reverse primers were 5'-ATA GTG TGG TTT ATG GAC TGA GGT C-3', 5'-CTT CTT CCTTAC TTT TGG GGT CTA A-3' respectively. For parvalbumin the forward and the reverse primers were $5^{\prime}-C C G$ AGT TGC AGG ATG TCG ATG A-3', 5'-GTG GAA GAC CAG GGG CAG TCA GT-3'. For ninjurin the forward and the reverse primers were $5^{\prime}-A A C$ CAT TAC GCC AGC AAG AAG AG-3', 5'-GTG ATG AAG ATG TTG ACT ACC A$3^{\prime}$. For VDR the forward and the reverse primers were $5^{\prime}$-TAA GAC CTA CGA CCC CAC CTA C-3', 5'-ATG GCA CTT GAC TTC AGC AGT $A-3^{\prime}$. For GAPDH the forward and the reverse primers were $5^{\prime}-G G A$ GCC AAA AGG GTC ATC ATC TCT G-3', 5'- TTT CTA GAC GGC AGG TCA GGT CCA C-3'. For proteinase 3 the forward and the reverse primers were $5^{\prime}$-CGT GAA GAA GTC AGG GAA AAG G-3' 5'ACT ACG ACG GCC AGA ACA AAC T-3'. For IL-8 the forward and the reverse primers were 5'-CTC TTG CGA GCC TTC CTG ATT TCT G-3', 5'-GCA ACC CTA CAA CAG ACC CAC ACA A-3'. For cyp24 the forward and the reverse primers were 5 -CCC GAG ACT GGT GAC ATC TAC-3', 5'-CGT AGC CTT CTT TGC GGT AGT-3'.

\section{Acknowledgements}

The authors gratefully acknowledge the generous support of the 'Cassa di Risparmio Foundation of Modena' for the acquisition of the 'Affymetrix System'. This work was supported by AlL (Italian Association against Leukemia), AIRC (Italian Association for Cancer Research), CIB (Italian Consortium for Biotechnology) and MURST-2000. 


\section{References}

1. Bennett JM, Catovsky D, Daniel MT, Flandrin G, Galton DA, Gralnick HR and Sultan C (1976) Proposals for the classification of the acute leukaemias. FrenchAmerican-British (FAB) co-operative group. Br. J. Haematol. 33: 451-458

2. Drexler HG. (1987) Classification of acute myeloid leukemias - a comparison of FAB and immunophenotyping. Leukemia 1: 697-705

3. Zeleznikle NJ, Nucifora G and Rowley JD (1995) The molecular biology of myeloproliferative disorders as revealed by chromosomal abnormalities [review]. Seminars in Hematology 32: 201-219

4. Ferrari S, Manfredini R, Grande A, Torelli G and Torelli U (1992) Proliferation, differentiation arrest, and survival in leukemic blast cells. Ann. N.Y. Acad. Sci. 663: $202-214$

5. Stoffel R, Ziegler S, Ghilardi N, Ledermann B, de Sauvage FJ and Skoda RC (1999) Permissive role of thrombopoietin and granulocyte colony-stimulating factor receptors in hematopoietic cell fate decisions in vivo. Proc. Natl. Acad. Sci. U.S.A $96:$ : $698-702$

6. Socolovsky M, Lodish HF and Daley GQ (1998) Control of hematopoietic differentiation: lack of specificity in signaling by cytokine receptors. Proc. Natl. Acad. Sci. USA 95: 6573-6575

7. Miller CW and Koeffler HP. (1990) In vivo and in vitro differentiation of myeloid leukemic cells. In The biology of human leukemia. Mauer AM ed Baltimore and London: The John Hopkins University Press

8. Breitman TR, Selonick SE and Collins SJ (1980) Induction of differentiation of the human promyelocytic leukemia cell line $(\mathrm{HL}-60)$ by retinoic acid. Proc. Natl. Acad. Sci. USA 77: 2936-2940

9. Castaigne S, Chomienne C, Daniel MT, Ballerini P, Berger R, Fenaux P and Degos $L$ (1990) All-trans retinoic acid as a differentiation therapy for acute promyelocytic leukemia: clinical results. Blood 76: 1704-1709

10. Studzinski GP, McLane JA and Uskokovic MR (1993) Signaling pathways for vitamin D-induced differentiation: implications for therapy of proliferative and neoplastic diseases. Crit. Rev. Eukaryot. Gene Expr. 3: 279-312

11. Manfredini R, Trevisan F, Grande A, Tagliafico E, Montanari M, Lemoli R, Visani $G$, Tura S and Ferrari S (1999) Induction of a functional vitamin $D$ receptor in alltrans-retinoic acid-induced monocytic differentiation of M2-type leukemic blast cells. Cancer Res. 59: 3803-3811

12. Lipshutz RJ, Fodor SP, Gingeras TR and Lockhart DJ (1999) High density synthetic oligonucleotide arrays. Nat. Genet. 21: 20-24

13. Schulze A and Downward J (2001) Navigating gene expression using microarrays - a technology review. Nat. Cell Biol. 3: E190-E195

14. Mills JC, Roth KA, Cagan RL and Gordon JI (2001) DNA microarrays and beyond: completing the journey from tissue to cell. Nat. Cell Biol. 3: E175-E178

15. Ashburner M, Ball CA, Blake JA, Botstein D, Butler H, Cherry JM, Davis AP, Dolinski K, Dwight SS, Eppig JT, Harris MA, Hill DP, Issel-Tarver L, Kasarskis A, Lewis S, Matese JC, Richardson JE, Ringwald M, Rubin GM and Sherlock G (2001) Creating the gene ontology resource: design and implementation. Genome Res. 11: 1425-1433
16. Ashburner M, Ball CA, Blake JA, Botstein D, Butler H, Cherry JM, Davis AP, Dolinski K, Dwight SS, Eppig JT, Harris MA, Hill DP, Issel-Tarver L, Kasarskis A Lewis S, Matese JC, Richardson JE, Ringwald M, Rubin GM and Sherlock G (2000) Gene ontology: tool for the unification of biology. The Gene Ontology Consortium. Nat. Genet. 25: 25-29

17. Hashimoto S, Suzuki T, Dong HY, Nagai S, Yamazaki N and Matsushima K (1999) Serial analysis of gene expression in human monocyte-derived dendritic cells. Blood 94: 845-852

18. Hashimoto SI, Suzuki T, Nagai S, Yamashita T, Toyoda N and Matsushima K (2000) Identification of genes specifically expressed in human activated and mature dendritic cells through serial analysis of gene expression. Blood 96: 2206-2214

19. Seo J, Kim M and Kim J (2000) Identification of novel genes differentially expressed in PMA-induced HL-60 cells using cDNA microarrays. Mol. Cells 10: 733-739

20. Pols HA, Birkenhager JC, Foekens JA and van Leeuwen JP (1990) Vitamin D: a modulator of cell proliferation and differentiation. J. Steroid Biochem. Mol. Biol. 37: $873-876$

21. Kelsey SM, Makin HL and Newland AC (1992) Functional significance of induction of differentiation in human myeloid leukaemic blasts by 1,25 dihydroxyvitamin D3 and GM-CSF. Leuk. Res. 16: 427-434

22. Le Naour F, Hohenkirk L, Grolleau A, Misek DE, Lescure P, Geiger JD, Hanash S and Beretta $L$ (2001) Profiling changes in gene expression during differentiation and maturation of monocyte-derived dendritic cells using both oligonucleotide microarrays and proteomics. J. Biol. Chem. 276: 17920-17931

23. Berer A, Stockl J, Majdic O, Wagner T, Kollars M, Lechner K, Geissler K and Oehler L (2000) 1,25-Dihydroxyvitamin D(3) inhibits dendritic cell differentiation and maturation in vitro. Exp. Hematol. 28: $575-583$

24. Tokuda N and Levy RB (1996) 1,25-dihydroxyvitamin D3 stimulates phagocytosis but suppresses HLA-DR and CD13 antigen expression in human mononuclear phagocytes. Proc. Soc. Exp. Biol. Med. 211: 244-250

25. Grande A, Montanari M, Tagliafico E, Manfredini R, Marani TZ, Siena M, Tenedin E, Gallinelli A and Ferrari S (2002) Physiological levels of 1alpha, 25 dihydroxyvitamin D3 induce the monocytic commitment of CD34+ hematopoietic progenitors. J. Leukoc. Biol. 71: 641-651

26. Collins SJ (1987) The HL-60 promyelocytic leukemia cell line: proliferation, differentiation, and cellular oncogene expression. Blood 70: 1233-1244

27. Manfredini R, Balestri R, Tagliafico E, Trevisan F, Pizzanelli M, Grande A, Barbieri D, Zucchini P, Citro G, Franceschi C and Ferrari S (1997) Antisense inhibition of c-fes proto-oncogene blocks PMA-induced macrophage differentiation in HL60 and in FDC-P1/MAC-11 cells. Blood 89: 135-145

28. MacDonald RJ, Swift GH, Przybyla AE and Chirgwin JM (1987) Isolation of RNA using guanidinium salts. Methods Enzymol. 152: 219-227 